\title{
Case Report: Remediating Cognitive Impairments in Dementia of Alzhimer's Type Through Concurrent Transcranial Direct Current Stimulation and Cognitive Training:A Case Report
}

\author{
Sanaz Tajadini ${ }^{1}\left(\mathbb{D}\right.$, Neda Mohammadi $^{1,2} \mathbb{D}$, Mahshid Tahamtan $^{1,2 *} \mathbb{C}$, Mohammad Nami $^{1,2,3}(\mathbb{C}$
}

1. Department of Neuroscience, School of Advanced Medical Sciences and Technologies, Shiraz University of Medical Sciences, Shiraz, Iran.

2. DANA Brain Health Institute, Shiraz, Iran.

3. Clinical Neurology Research Center, Shiraz University of Medical Sciences, Shiraz, Iran.

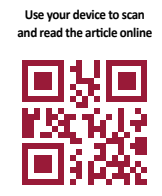

Citation: Tajadini S, Mohammadi N, Tahamtan .M, Nami M. Remediating Cognitive Impairments in Alzheimer Type Though Concurrent Transcranial Direct Current Stimulation and Cognitive Training: A Case Report. JAMSAT. 2017; 3(4):227-234. https:// doi.org/10.32598/jamsat.3.4.227

doif $h$ htps://doi.org/10.32598/jamsat.3.4.227

Article info:

Received: 08 May 2017

Accepted: 13 Aug 2017

Keywords:

Dementia of alzheimer type, Cognitive impairment, Transcranial direct current stimulation

\begin{abstract}
A B STRACT
Objectives: Dementia of Alzheimer Type (DAT) is associated with progressive cognitive impairments. Such a clinically significant condition is known to affect approximately $9.5 \%$ of people over 70 years of age. However, it is accepted as a more challengeable medical entity because of its increasing atypical presentation, rarity of efficient treatments, and diagnostic and prevention challenges.
\end{abstract}

Materials \& Methods: we present a case who referred with 5 months history of cognitive decline following Medial Temporal Lobe (MTL) atrophy upon neuroimaging. Following cognitive and neurophysiological assessments, she underwent 21 consecutive sessions of transcranial Direct Current Stimulation (tDCS) 3 times per week. Concurrently, a media-rich computer platform was administered for cognitive and behavioral remediation.

Results: Based on the evidence regarding the use of tDCS in dementia, we aimed at stabilizing the cognitive profile and halting or slowing down the process of progressive cognitive decline in the present case. The outcome of our neuromodulatory intervention using tDCS supported the beneficial impact of such an approach in not only stabilizing but also ameliorating cognitive functions. This is especially important when rapid progression of cognitive symptoms in DAT is of concern.

Conclusion: The promising clinical course of this specific case supports the possible beneficial effects of tDCS in halting the progression of symptoms in DAT. Sham-controlled clinical trials would get momentum to highlight clinical impact of such an intervention in DAT.

\section{* Corresponding Author:}

Address: Department of Neuroscience, School of Advanced Medical Sciences and Technologies, Shiraz University of Medical Sciences, Shiraz, Iran. Tel: +98 (71) 32305471

E-mail:mahshidtahamtan@yahoo.com 


\section{Introduction}

D

ementia of Alzheimer Type (DAT) is a ubiquitous neurodegenerative process and the most common cause of dementia in the elderly, with substantial cost burden on individuals and the society [1]. It is associated with senility and affect approximately $9.5 \%$ of people older than 70 years [2]. Regarding its adverse effects on the quality of life, life expectancy, and societal costs, the World Alzheimer Report and World Health Organization recommend dementia care as a public health priority [3] .

DAT is commonly associated with Mild Cognitive Impairment (MCl), which may contribute to the decline in daily functioning, learning, executive functions, and contribute to the minor linguistic problems, loss of memory and attention deficits [4]. $\mathrm{MCl}$ results from actual damage to lateral frontoparietal and medial temporal regions. This damage can lead to defects in learning new information and retrieving old memories [5]; however, depression, anxiety and irritability may also manifest in DAT patients [6]

Therefore, lack of effective interventions have diverted the attention toward successful therapeutic strategies as a major goal in health interventions in order to improve or prevent DAT [7] . In this regard, as a new noninvasive and neuro-modulatory technique, transcranial Direct Current Stimulation (tDCS) has been widely used in DAT individuals to improve their cognitive capacities [8], and alter brain activity in specific regions with its weak direct current over the patients' scalps [9].

Based on clinical practice of tDCS in patients with DAT, it can improve cognitive functions, due to its ability in altering excitability of brain cells [10-12]. However, a few studies have reported negative results $[13,14]$. A reasonable interpretation would be due to montage used, different participants, or the method of evaluation. To this end, we present a woman suffering from DAT with cognitive decline. We present this case discussion to gain more insight over online neuro-modulatory (tDCS) effects on stabilizing the cognitive decline in patient with DAT.

\section{Case Report}

\section{Patient's information}

The patient was a 55-year-old woman, housewife, and native Persian speaker with 5 years formal education. She presented to our Neuroscience Laboratory with a 5-month history of cognitive decline following Medial Temporal Lobe (MTL) atrophy. Based on her daughter's report, she frequently repeated herself, distracted and failed to connect with others. However, her main complaints were recent weakening in working memory, attention, executive functions, and language reconstruction. She had dysarthria and poverty of speech.

\section{Clinical findings and diagnostic assessment}

According to patient's Quantitative Electroencephalography (QEEG) rhythms based on frequency bands and functional cortical connectivity and neuropsychiatric assessments, the clinical diagnosis of DAT was confirmed. A 24-ch QEEG recording was administered, using the NR-sign amplifier through 10-20 system, in two phases. In phase one, data were recorded while the patient kept her eyes open in a relaxed state, without moving or talking. On examination, background activity was normal, but the patient exhibited lower power density in posterior alpha waves $(8-12 \mathrm{~Hz})$. Furthermore, FFT analysis showed increased widespread theta $(4-7 \mathrm{~Hz})$ rhythms in bi-temporal regions mainly on the left hemisphere.

On the second phase, during repetition verbal memory task, the results showed a coherent decrease in QEEG rhythms at F7-T5 electrodes in the beta band (13-30 Hz), and also lack of high beta power density in frontopolar brain regions. Finally higher power density of theta waves in left frontotemporal suggested verbal memory deficits (Figure 1).

The patient also underwent general cognitive assessment (Montreal Cognitive Assessment [MoCA]), as a measure of her general cognitive status; language, memory, attention, and executive function [15]. The results are presented in Table 1. Clinical symptoms, QEEG results, and below cutoff scores for normal performance on MoCA confirmed the proposed diagnosis for early-onset DAT.

\section{Therapeutic intervention}

\section{Experimental protocol}

After analyzing the patient's baseline performance and taking her consent, we recommended 21 tDCS sessions, 3 times per week, in combination with Cognitive Behavioral Therapy (CBT) and other behavioral online interventions. In the following, the different procedural phases for offline and online tDCS are shown in their temporal order (Figure 2).

\section{tDCS}

Two-channel tDCS was bilaterally delivered by a battery-driven current stimulator connected to 4 sponge 


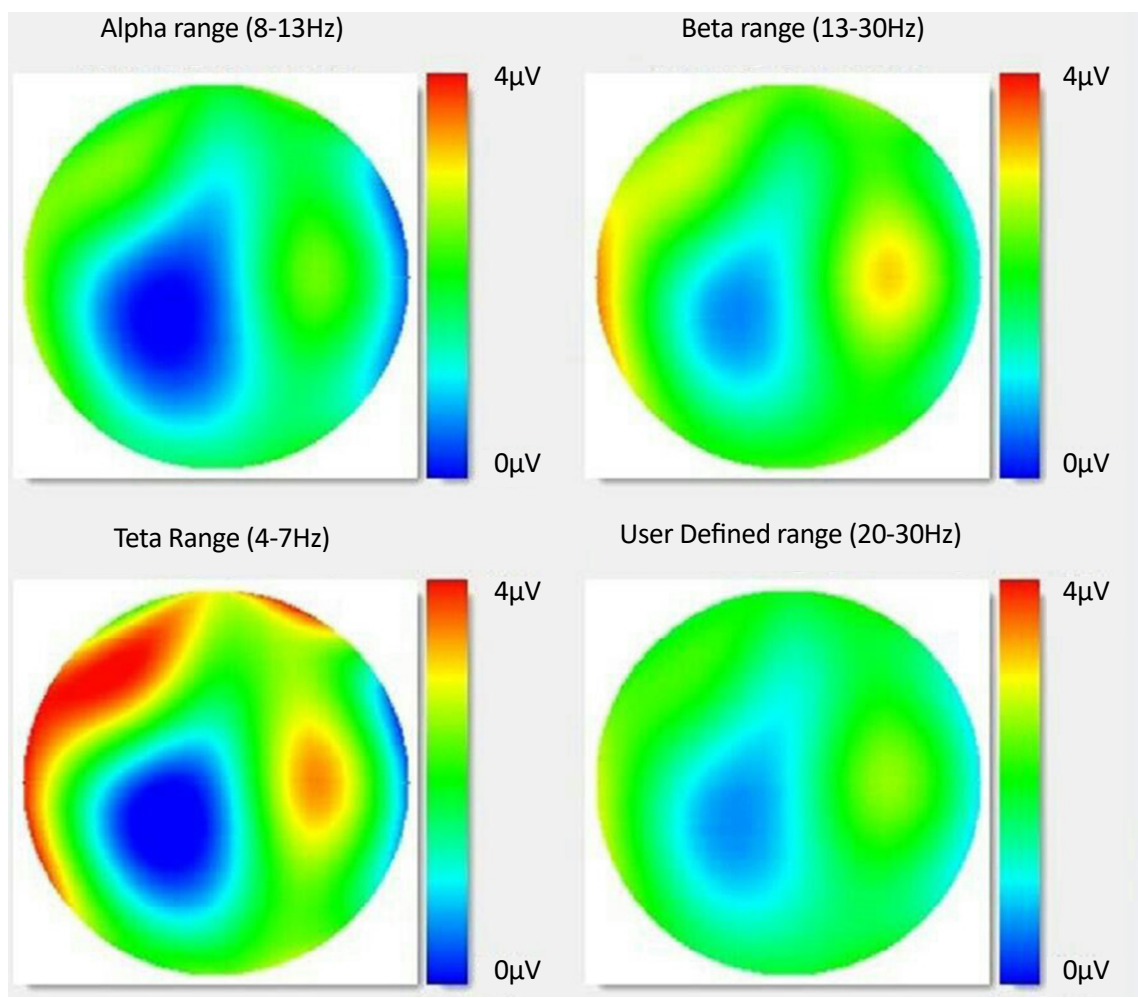

JAMSAT

Figure 1. The color-coded quantitative EEG brain map upon repetition verbal memory task. High power density of theta in left frontotemporal and lack of high beta power density in frontopolar brain regions were recognized

electrodes. We used the saline-soaked synthetic sponge for tDCS to minimize skin resistance. Two stimulation electrode (the anodes) sites were left temporal and prefrontal cortex, positioned over F3 and T3. Cathodal electrodes were placed over F4 and FP2 based on the 10-20 EEG international system. A constant current intensity of $2 \mathrm{~mA}$ was applied for 45 minutes for every session. During each session, there was a ramping period of $10 \mathrm{~s}$.

\section{Online tDCS experiments (cognitive assessments)}

To monitor prodromes and modify patient's behavior, we used four different tasks: Cognitive Behavioral Therapy (CBT), Rey Auditory Verbal Learning Test (RAVLT), Purdue pegboard test, and match-to-sample task. To screen the patient's explicit memory and short-term auditory-verbal memory impairments, CBT and RAVLT

Table 1. Score of the subject in some subtests of MoCA

\begin{tabular}{|c|c|c|c|}
\hline Tasks & Raw Score & Results & Cutoff \\
\hline Executive & 0.5 & Below & - \\
\hline Naming & 0.3 & Below & - \\
\hline Memory & 2.5 & Below & - \\
\hline Attention & 1.6 & Below & - \\
\hline Language & 1.3 & Below & - \\
\hline Abstractor & 0.2 & Below & - \\
\hline Orientation & 2.6 & Below & - \\
\hline Total & 6.30 & - & Normal>26 \\
\hline
\end{tabular}


Experinmental protocol
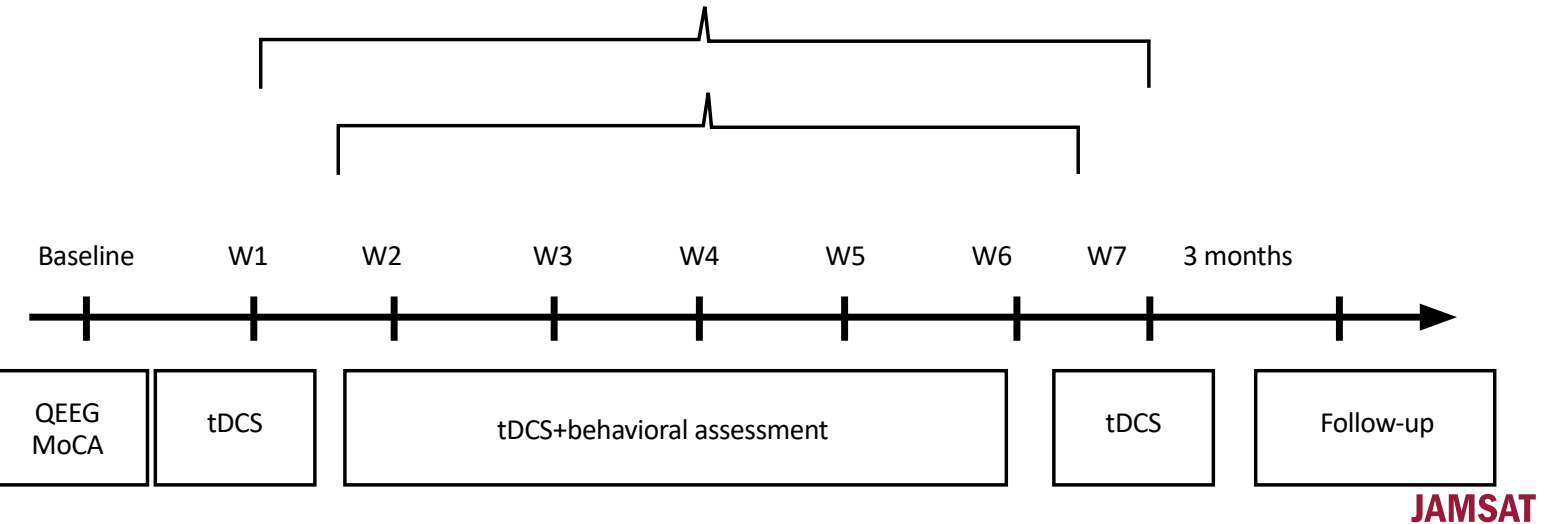

Figure 2. Experimental protocol for transcranial Direct Current Stimulation (tDCS). For preparing baseline information, QEEG and MoCA was done. tDCS was performed for 3 days a week, 7 weeks with or without behavioral assessment.

questionnaires were administered to obtain a stable online experimental phase, in which the patient was asked to recall more positive and neutral words and also repeat different word trials after hearing them.

The patient's eye-hand coordination, gross muscle coordination of the arms and hands, and fine motor coordination of the fingers were assessed using uniand bi-manual dexterity (pegboard). Visual matching ability was assessed with short-term visual recognition memory task (match-to-sample). In general, based on pegboard instruction, three separate sections were administered: right hand alone; left hand alone; and both hands. The mean number of pegs the patient placed in the board for 3 consecutive trials (bar, ring, and cylinder) served as the test score. The standard administration lasted $30 \mathrm{~s}$ per trial. The match-tosample task comprised three successive levels which in each level the patient required to remember, identify, and push the shapes to match stimuli. Follow-up visit was recommended.

\section{Results}

Here, we report the results of different analysis to certify the clinical conditions of the present case report. The patient attended all experimental sessions. After completing the clinical trials, the patient in both CBT and RAVLT tests showed stable functioning during stimulation condition ( 7.10 and 15.15 points, respectively). Of note, these procedures were repeated for two sessions after the first week of offline tDCS. During the 5 -session period, the pa-

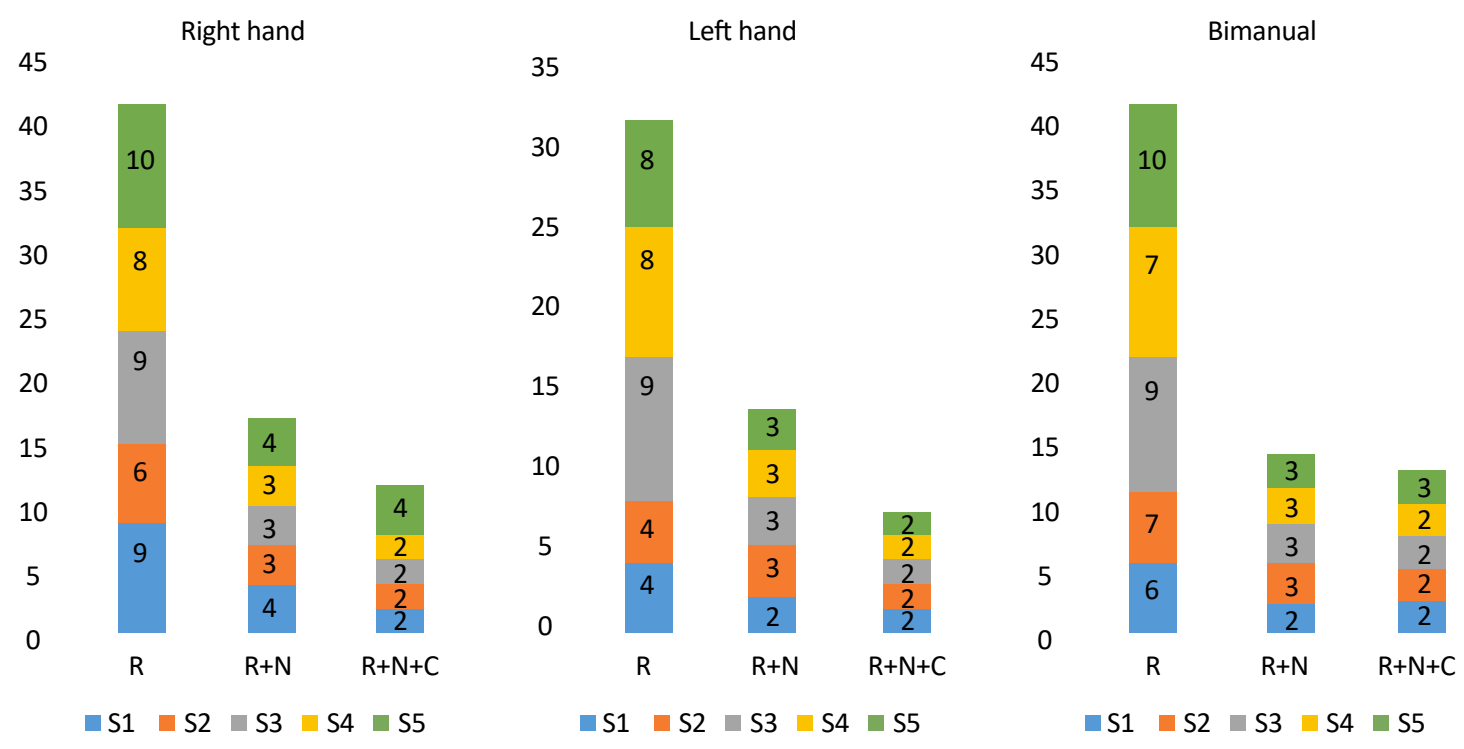

JAMSAT

Figure 3. The pegboard analysis in five sessions for three sections. The patient's sensorimotor function improved in each section. 


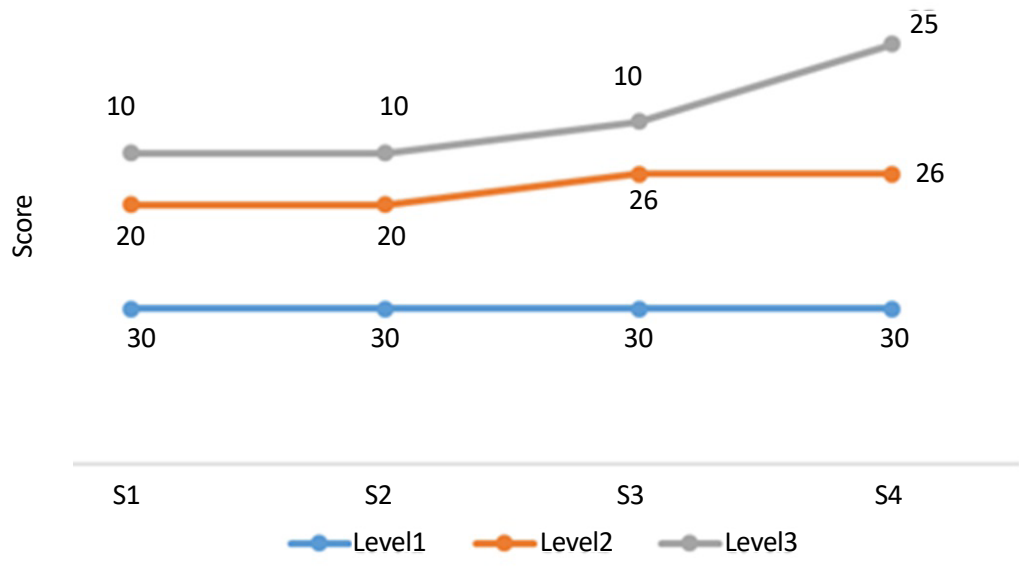

JAMSAT

Figure 4. The match-to-sample analysis in five sessions. There was an increasing trend in patient's performance during S3, S4 at level 2 and S4 at level 3.

tient's sensorimotor functions on pegboard task improved in each section (Figure 3). For the other online cognitive experiment, the performance on match-to-sample task was analyzed; one-week patient's attention and visual recognition memory was observed during S1, S2 at level 2 and S1, S2, and S3 at level 3. In accordance with our expec- tations, there was an increasing trend in patient's performance during S3, S4 at level 2 and S4 at level 3 (Figure 4).

\section{Treatment and follow-up}

Following the neurostimulation and neuropsychological procedures, improvement of cognitive functions

Table 2. Non-invasive stimulation studies targeting cognitive and neuropsychiatric symptoms in dementia

\section{Study Patient Group Treatment Stimulation Parameters Outcome Measures Results}

\begin{tabular}{|c|c|c|c|c|}
\hline $\begin{array}{l}\text { AD }(n=10 ; \\
\text { NINCDS-ARDA cri- } \\
\text { teria with MMSE } \\
\text { of } 12-25)\end{array}$ & tDCS & $\begin{array}{l}\text { Active ( } 2 \text { mA for } 30 \text { min- } \\
\text { utes) or sham ( } 2 \text { mA for } 30 \\
\text { seconds) stimulation ( } 35 \\
\mathrm{~cm}^{2} \text { electrodes). Anode } \\
\text { placed on left DLPFC (F3) } \\
\text { or left temporal cortex } \\
\text { (T7) with cathode on right } \\
\text { supraorbital area. } \\
\text { Left DLPFC, left temporal } \\
\text { cortex and sham stimula- } \\
\text { tion given in counterbal- } \\
\text { anced manner with } 48 \\
\text { hours washout period } \\
\text { between each condition. }\end{array}$ & $\begin{array}{c}\text { Selective attention } \\
\text { (stroop), working } \\
\text { memory (backward } \\
\text { and forward digit span) } \\
\text { and visual recognition } \\
\text { memory tasks }\end{array}$ & $\begin{array}{l}\text { Improvement shown in visual } \\
\text { recognition memory task after } \\
\text { active stimulation (approx. 18\% } \\
\text { improvement after temporal and } \\
\text { 14\% after prefrontal stimulation) } \\
\text { compared to sham. }\end{array}$ \\
\hline
\end{tabular}

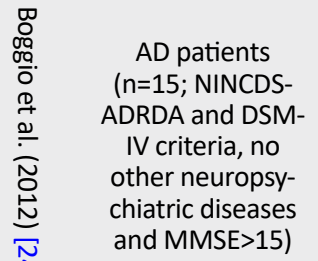

tDCS

Active or sham anodal stimulation ( $2 \mathrm{~mA}$ for 30 $\mathrm{min})$ delivered to T3 and T4 bilaterally $\left(35 \mathrm{~cm}^{2}\right.$ electrodes with $64 \mathrm{~cm}^{2}$ electrode placed over right deltoid muscle) for 5 consecutive days.
Improvement shown on visual recognition task compared to

MMSE, ADAS-Cog, visual recognition and visual attention tasks with improvements sustained at 1 month follow-up period (11.4\% improvement). No effects shown on MMSE, ADAS-cog or visual attention. 


\section{Study Patient Group Treatment Stimulation Parameters Outcome Measures Results}

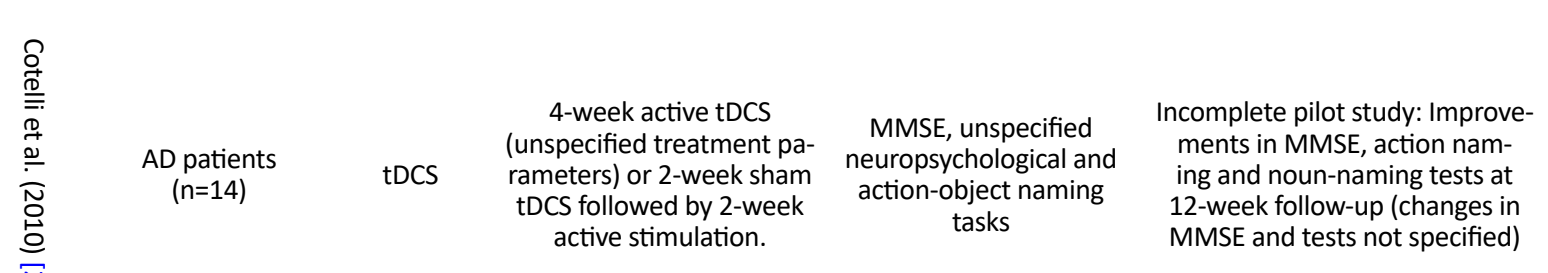

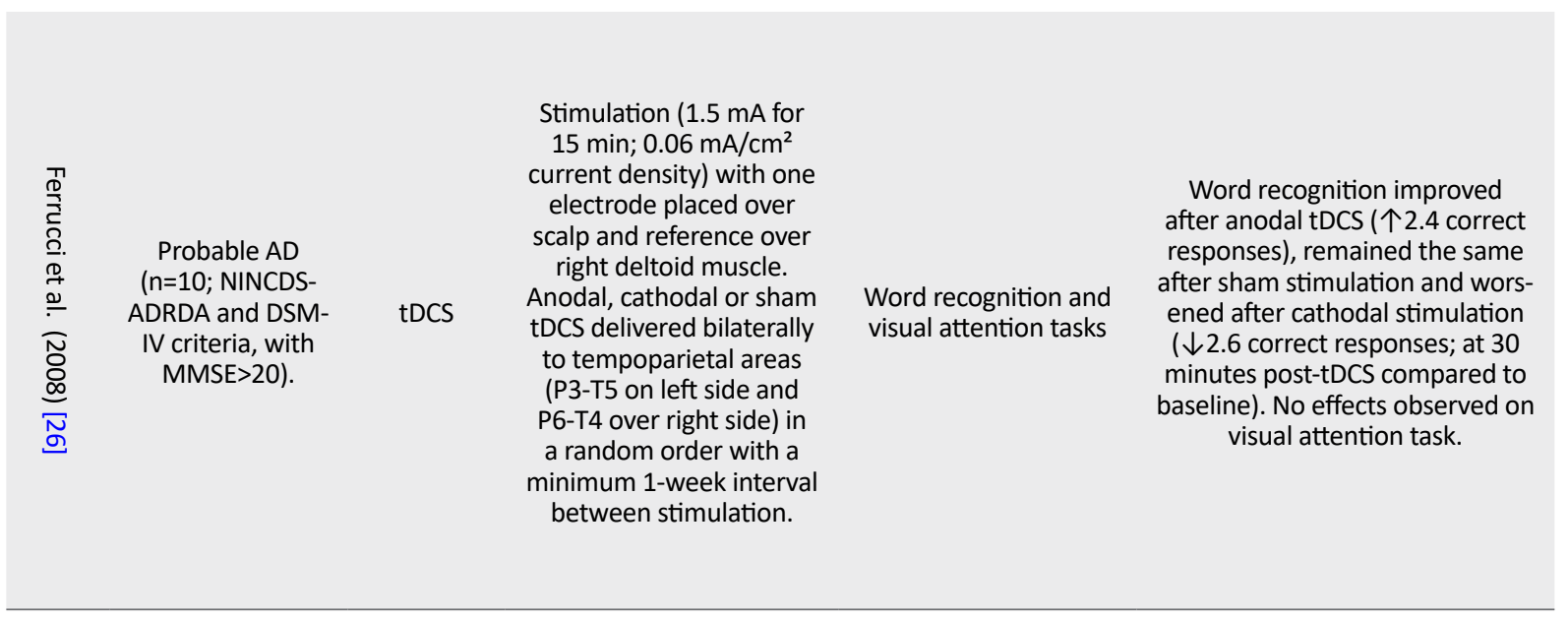

JAMSAT

persisted 3 months after tDCS application. To keep the patient's cognitive functions, she was advised to stop her previous medications and switch to piracetam, donepezil, Ritalin, neurazol, and vitamin E. The follow-up evaluation in our laboratory suggested a notable subjective improvement in her social scale quality and her family members reported significant behavior changes like her reducing anxiety, aggression, and depression, and also improvement in speech fluency.

\section{Discussion}

Based on the previous studies, Alzheimer Disease (AD) is the most common cause of dementia. It also determines the loss of cognitive functioning with increasing age [16, 17]. The worsening clinical situation of the patient in addition to the degree of impairments in cognitive function forced us to perform stimulation technique. Suggesting the positive role of tDCS on cognitive deficits, there have been only a few case reports in which benefits were realized by consecutive sessions of tDCS applied over the temporal and frontal areas $[18,19]$. This report therefore extends previous findings by showing significant improvement in learning and recognition memory via concurrent delivery of tDCS [20, 21]. Accordingly, from our experience with this patient, we tend to agree with the results from a comprehensive literature search on tDCS studies in AD subjects until March 2017 in which 12 articles with a total of 202 AD participants demonstrated positive results with tDCS regarding their improvement in cognition [22]. Moreover, Table 2 presents details regarding the information related to the tDCS effects in four studies [23-26]. Generally, despite the well-known positive effects of tDCS as an adjuvant treatment for patients with $A D$, the conclusion of this report is limited due to the results of a single case. However, it was important for us to share this unique patient as a case report who experienced multi-cognitive impairments under brain stimulation and various cognition assessments in different domains. However on the basis of clinical features, parameters such as duration and intensity of stimulation are as casual factors underlying tDCS effects [27]. We hope that by reporting this case, further studies involving newer techniques like high-definition tDCS and multimodal neuroimaging investigations were carried out that can have important implications for clinical situation regarding neural basis of tDCS effects in DAT. 


\section{Ethical Considerations}

\section{Compliance with ethical guidelines}

The informed consent was obtained from the subject declaring that his clinical data would be used in our institutional research database.

Funding

This research did not receive any specific grant from funding agencies in the public, commercial, or not-forprofit sectors.

Conflict of interest

The authors declared no conflict of interest.

\section{References}

[1] Sosa Ortiz AL, Acosta Castillo I, Prince MJ. Epidemiology of dementias and Alzheimer's disease. Archives of Medical Research. 2012; 43(8):600-8. [DOI:10.1016/j.arcmed.2012.11.003] [PMID]

[2] Brookmeyer R, Evans DA, Hebert L, Langa KM, Heeringa SG, Plassman BL, et al. National estimates of the prevalence of Alzheimer's disease in the United States. Alzheimer's \& Dementia. 2011; 7(1):61-73. [DOI:10.1016/j.jalz.2010.11.007]

[3] World Health Organization. Dementia: A public health priority Geneva: World Health Organization; 2012.

[4] Alzheimer's Disease International: The Global Voice and dementia. World Alzheimer report 2015: The global impact of dementia. London: Alzheimer's Disease International; 2015.

[5] Bäckman L, Small BJ, Fratiglioni L. Stability of the preclinical episodic memory deficit in Alzheimer's disease. Brain. 2001; 124(1):96-102. [DOI:10.1093/brain/124.1.96] [PMID]

[6] Petersen RC, Caracciolo B, Brayne C, Gauthier S, Jelic V, Fratiglioni L. Mild cognitive impairment: A concept in evolution. Journal of Internal Medicine. 2014; 275(3):214-28. [DOI:10.1111/ joim.12190] [PMID] [PMCID]

[7] Robinson L, Tang E, Taylor JP. Dementia: timely diagnosis and early intervention. BMJ. 2015; 350:h3029.[DOI:10.1136/bmj.h3029] [PMID] [PMCID]

[8] Hill AT, Fitzgerald PB, Hoy KE. Effects of anodal transcranial direct current stimulation on working memory: A systematic review and meta-analysis of findings from healthy and neuropsychiatric populations. Brain Stimulation. 2016; 9(2):197-208. [DOI:10.1016/j. brs.2015.10.006

[9] Stagg CJ, Nitsche MA. Physiological basis of transcranial direct current stimulation. The Neuroscientist. 2011; 17(1):37-53. [DOI:10.1177/1073858410386614] [PMID]

[10] Elder GJ, Taylor JP. Transcranial magnetic stimulation and transcranial direct current stimulation: treatments for cognitive and neuropsychiatric symptoms in the neurodegenerative dementias. Alzheimer's Research \& Therapy. 2014; 6(5):74. [DOI:10.1186/ s13195-014-0074-1] [PMID] [PMCID]

[11] Meinzer M, Lindenberg R, Phan MT, Ulm L, Volk C, Flöel A. Transcranial direct current stimulation in mild cognitive impairment: Behavioral effects and neural mechanisms. Alzheimer's \& Dementia. 2015; 11(9):1032-40. [DOI:10.1016/j.jalz.2014.07.159]

[12] Yun K, Song IU, Chung YA. Changes in cerebral glucose metabolism after 3 weeks of noninvasive electrical stimulation of mild cognitive impairment patients. Alzheimer's Research \& Therapy. 2016; 8(1):49. [DOI:10.1186/s13195-016-0218-6] [PMID] [PMCID]

[13] Cotelli M, Manenti R, Brambilla M, Petesi M, Rosini S, Ferrari C, et al. Anodal tDCS during face-name associations memory training in Alzheimer's patients. Frontiers in Aging Neuroscience. 2014; 6:38. [DOI:10.3389/fnagi.2014.00038] [PMID] [PMCID]

[14] Bystad M, Grønli O, Rasmussen ID, Gundersen N, Nordvang L, Wang Iversen $\mathrm{H}$, et al. Transcranial direct current stimulation as a memory enhancer in patients with Alzheimer's disease: A randomized, placebo-controlled trial. Alzheimer's Research \& Therapy. 2016; 8(1):13. [DOI:10.1186/s13195-016-0180-3] [PMID] [PMCID]

[15] Nasreddine ZS, Phillips NA, Bédirian V, Charbonneau S, Whitehead V, Collin I, et al. The montreal cognitive assessment, MoCA A brief screening tool for mild cognitive impairment. Journal of the American Geriatrics Society. 2005; 53(4):695-9.[DOI:10.1111/ j.1532-5415.2005.53221.x] [PMID]

[16] Brookmeyer R, Johnson E, Ziegler Graham K, Arrighi HM. Forecasting the global burden of Alzheimer's disease. Alzheimer's \& Dementia. 2007; 3(3):186-91. [DOI:10.1016/j.jalz.2007.04.381]

[17] Bernick C, Cummings J, Raman R, Sun X, Aisen P. Age and rate of cognitive decline in Alzheimer disease: Implications for clinical trials. Archives of Neurology. 2012; 69(7):901-5. [DOI:10.1001/ archneurol.2011.3758] [PMID]

[18] Bystad M, Rasmussen ID, Grønli O, Aslaksen PM. Can 8 months of daily tDCS application slow the cognitive decline in Alzheimer's disease? A case study. Neurocase. 2017; 23(2):146-8. [DOI:10.108 0/13554794.2017.1325911] [PMID]

[19] Costa V, Brighina F, Piccoli T, Realmuto S, Fierro B. Anodal transcranial direct current stimulation over the right hemisphere improves auditory comprehension in a case of dementia. NeuroRehabilitation. 2017; 41(2):567-75. [DOI:10.3233/NRE-162062] [PMID]

[20] Hampstead BM, Sathian K, Bikson M, Stringer AY. Combined mnemonic strategy training and high-definition transcranial direct current stimulation for memory deficits in mild cognitive impairment. Alzheimer's \& Dementia: Translational Research \& Clinica Interventions. 2017; 3(3):459-70. [DOI:10.1016/j.trci.2017.04.008] [PMID] [PMCID]

[21] Roncero C, Kniefel H, Thiel A, Probst S, Chertkow H. Inferior parietal transcranial direct current stimulation with training im proves cognition in anomic Alzheimer's disease and frontotem poral dementia. Alzheimer's \& Dementia: Translational Research \& Clinical Interventions. 2017; 3(2):247-53. [DOI:10.1016/j trci.2017.03.003] [PMID] [PMCID]

[22] Liu CS, Rau A, Gallagher D, Rajji TK, Lanctôt KL, Herrmann N. Using transcranial direct current stimulation to treat symptoms in mild cognitive impairment and Alzheimer's disease. Neurodegenerative Disease Management. 2017; 7(5):317-29. [DOI:10.2217/ nmt-2017-0021] [PMID] 
[23] Boggio PS, Khoury LP, Martins DC, Martins OE, Macedo EC, Fregni F. Temporal cortex DC stimulation enhances performance on a visual recognition memory task in Alzheimer's disease. Journal of Neurology, Neurosurgery \& Psychiatry. 2008; 80(4):444-7. [DOI:10.1136/jnnp.2007.141853] [PMID]

[24] Boggio PS, Ferrucci R, Mameli F, Martins D, Martins O, Vergari $M$, et al. Prolonged visual memory enhancement after direct current stimulation in Alzheimer's disease. Brain Stimulation. 2012; 5(3):223-30. [DOI:10.1016/j.brs.2011.06.006]

[25] Cotelli M, Manentil R, Rosini S, Brambilla M, Zanetti O, Miniussi C. Brain stimulation in Alzheimer disease. Neuropsychol Trends. 2010; 8:57-60.

[26] Ferrucci R, Mameli F, Guidi I, Mrakic-Sposta S, Vergari M, Marceglia S, et al. Transcranial direct current stimulation improves recognition memory in Alzheimer disease. Neurology. 2008; 71(7):493-8. [DOI:10.1212/01.wnl.0000317060.43722.a3] [PMID]

[27] Zhao H, Qiao L, Fan D, Zhang S, Turel O, Li Y, et al. Modulation of brain activity with noninvasive transcranial Direct Current Stimulation (tDCS): Clinical applications and safety concerns. Frontiers in Psychology. 2017; 8:685. [DOI:10.3389/fpsyg.2017.00685] [PMID] [PMCID] 